\title{
A Rose By Any Other Name?
}

Richard Marlin, PhD

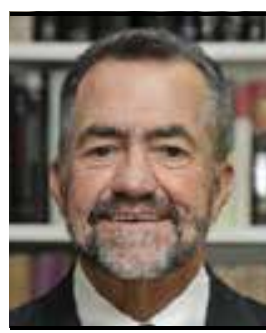

\begin{abstract}
About the Author
Dr. Richard Marlin is a clinical psychologist with more than 25 years of experience treating and consulting with industry about chronic disability problems including various chronic pain syndromes, chronic fatigue syndromes and fibromyalgia. He also treats patients with such problems as chronic depression and anxiety. Dr. Marlin is currently the director of Odyssey Health Services.
\end{abstract}

$I^{\prime}$ n February of this year, an expert committee of the United States Institute of Medicine (IOM) released a lengthy report in which its members reviewed diagnostic criteria and proposed a new label for chronic fatigue syndrome, also historically referred to as myalgic encephalomyelitis. ${ }^{1}$ The committee's proposed new label for this illness is Systemic Exertion Intolerance Disease. The report refers to the fact that a sizeable population is diagnosed with this illness, which causes considerable suffering and functional impairment. Many patients also feel stigmatized because of the label chronic fatigue syndrome.

An editorial was published in The Lancet in the same month $^{2}$ that supported the proposal for the inclusion of a broader array of symptoms in patients with the diagnosis, including: impaired day-to-day functioning secondary to fatigue, malaise after exertion, and unrefreshing sleep as well as cognitive difficulties or orthostatic intolerance, or both.

The notion of including exertion intolerance is reasonable and is more inclusive than the term 'chronic fatigue,' but it should also be acknowledged that fatigue at rest is a prominent symptom in the majority of patients. Of more concern is whether the use of the term 'disease' is valid and whether it will do more harm than good. ${ }^{3}$

The Institute of Medicine's report clearly acknowledges that no underlying objectively definable disease process or pathology has been identified to account for the symptoms experienced by these patients. Indeed, consistent with earlier CDC diagnostic criteria, any known definable disease or pathology that could account for the patient's symptoms must be ruled out before such a diagnosis can be made.

It appears that the utilization of the term disease is intended to both convey the severity and the reality of patients' suffering and associated impaired functioning. ${ }^{4}$
I agree that the suffering and impaired functioning of these patients should be both acknowledged and validated. I also agree that the suffering that they experience should not be trivialized. However, the notion that labeling their suffering and impaired function as a disease will actually serve to reduce stigmatization and improve patient care is open to discussion.

In a broader healthcare context, we have, for some time, been battling the stigmatization of patients with what are broadly referred to as mental health difficulties. There has been a general cultural perception, which extends into the healthcare community, that psychological, emotional or behavioural difficulties are somehow less important than physical health difficulties. While considerable efforts have been undertaken to alter this perception in recent years, the effectiveness of these efforts themselves are often mixed.

From my perspective, efforts that directly and openly validate the reality of psychological suffering and the fact that the presence of such suffering can markedly restrict individuals' lives is important and needs to be emphasized. To attribute and treat the presence of such difficulties as a character flaw or a simplistic inadequacy on the part of the patient is demeaning, erroneous and harmful. The fact is that psychological suffering can be extremely painful, provoke enormous distress and wreak havoc with people's ability to carry on a meaningful life. To be fully aware of, and accept, the true nature of this suffering is a necessary requirement to both provide and to receive therapeutic assistance.

Attempts have been made to destigmatize mental health patients' symptoms by proposing that they represent neurochemical imbalances and/or diseased or pathological brain structure. This is despite the absence of convincing scientific evidence that such brain pathophysiology is a cause of these health problems. 
By suggesting that patients are better off if we tell them that their difficulties are caused by brain disease, we might be worsening the stigmatization of psychological suffering and impairment. Instead, I feel that we should accept these difficulties for what they are: very real human suffering in the psychological realm. Such acceptance will permit patients to acknowledge their suffering and distress and to seek help. Such help should include evidence-based psychological therapies, including behavioural therapies and cognitive behavioural therapies.

If patients believe their difficulties to be caused by brain disease, they are more likely to seek unproven medical solutions, including medication or interventions such as electroconvulsive therapy or transcranial magnetic therapy, the benefits of which are uncertain and controversial. It is well established that interventions used in studies funded by sponsoring drug companies consistently report better efficacy for drugs than studies with the same interventions that are not funded by drug companies. It has also been shown that when active placebos are used and the study is double-blind, the magnitude of effect is reduced.

I believe the same applies to patients suffering from Chronic Fatigue Syndrome/Systemic Exertional Intolerance. At the present time, cognitive behavioural therapy and carefully graded exercise are the only treatments with a solid evidence base. ${ }^{5}$ These interventions have produced significant improvement in patient's functional abilities, including improved exercise tolerance and return to gainful employment. Therefore, if appropriately addressed with evidence-based treatment, we can reduce both suffering and dysfunction in these patients. As an aside, these interventions have been shown to alleviate suffering even when we are dealing with definable disease and well-defined conditions. ${ }^{6}$ Thus, there is a considerable body of evidence that psychological, behavioural and psychosocial factors play a very important role in the degree to which patients become impaired and disabled and experience secondary suffering.

The notion of attaching the label 'disease' to these difficulties (when there is no credible scientific evidence of an identified disease process) carries the same risk as labeling what we typically call mental health difficulties as brain disease. Unfortunately, patients can be made to feel that their suffering is not real or disabling if they are offered psychological therapy. By labeling the condition as a disease both patients and healthcare practitioners are more likely to search for and utilize unproven biomedical interventions. Such effects will neither serve patients nor healthcare providers well.
The editorial in The Lancet notes the considerable number of 'unfair' criticisms directed at both the PACE trial' as well as the very recent Cochrane review which support the efficacy of graded exercise in the treatment of this illness. ${ }^{8}$ I would argue that this is a clear manifestation of the negative stigmatization of psychological and behavioural issues and treatment clearly directed at such issues.

There is a substantive body of scientific literature describing the role that such factors play in this and similar illnesses and, more importantly, in the degree of suffering that patients experience and the degree of reduced function. The psychological and behavioural factors include such fundamental processes as perceptual learning, conditioned physiological responses and operant conditioning. Quite understandably, beliefs and fears can play an important role in the how symptoms are perceived. ${ }^{9}$

If healthcare providers are hesitant to educate patients about such factors and fail to strongly recommend what is an evidence-based conceptualization of the illness and treatment, they are doing patients a considerable disservice. However, it is also essential that they validate the true degree of suffering and diminished function that the patient experiences.

To quote the editorial from The Lancet, "The message of the IOM report is that CFS/ME is a serious and complex disorder and the authors hope that the new name could be an important step in changing perception. Further research to test these new concepts with cooperation between professional and patient groups is now needed to improve the evidence base."

I concur that it is imperative that evidence be gathered to evaluate the impact of this document. One can hope that the net result is the removal of stigmatization with respect to this particular illness and the removal of the stigmatization of the contribution of psychological and behavioural factors and the stigmatization of effective behavioural treatment. Sadly, I am not optimistic that incorporating the term 'disease' will further that cause.

\section{References}

1. Fukuda K, Straus SE, Hickie I, Sharpe MC, Dobbins JG, Komaroff A. The chronic fatigue syndrome: a comprehensive approach to its definition and study. Annals of Internal Medicine 1994; 121:953-955.

2. Lancet. February 22, 2015; 385: 663.

3. See: Cott, A. The Disease-Illness Distinction: A Model for Effective and Practical Integration of Behavioural and Medical Science. In: McHugh, S. and Vallis, M. T. (Eds) Illness Behaviour: A Multidisciplinary Model. Plenum Press. 1986; 71-99; and Mechanic, D. The Concept of Illness Behavior. Journal of Chronic Disease 1961: 15; 189-94.

4. Whiting, P.; Bagnall, A.; Sowden, A., Cornell, J. ; Mulrow, C. and Ramirez, G. Interventions for the Treatment and Management of Chronic Fatigue Syndrome: a Systematic Review, Journal of the American Medical 


\section{A Rose By Any Other Name?}

Association, September 19, 2001, Volume 286, Number 11; Marlin, R., Anchel, H., Gibson, J., Goldberg, W. and Swinton, M. An evaluation of Multidisciplinary Intervention for Chronic Fatigue Syndrome with LongTerm Follow-Up and a Comparison with Untreated Controls. American Journal of Medicine, 1998: 105.(3A, 1105-1145 and Prins, P., van der Meer, J. and Bleijenberg, G. Chronic fatigue syndrome. Lancet January 28, 2006.

5. ACOEM Guideline: Preventing Needless Work Disability by Helping People Stay Employed. Journal of Occupational and Environmental Medicine 2006; 48, 9, 972-987.

6. White PD, Goldsmith KA, Johnson AL, Potts L, Walwyn R, DeCesare JC, et al. Comparison of adaptive pacing therapy, cognitive behaviour therapy, graded exercise therapy, and specialist medical care for chronic fatigue syndrome (PACE): a randomised trial. Lancet 2011; 377:82336;and Bleijenberg, G. and Knoop, H. Chronic Fatigue Syndrome: Where to PACE from here? Lancet. Published on-line February 18, 2011.

7. Larun L, Brurberg KG, Odgaard-Jensen J, Price JR. Exercise therapy for chronic fatigue syndrome. The Cochrane Library 2015, Issue 2.
8. See, for example: Nijs, J., Meeus, M., Van Oosterwijck,J., Ickmans,K., Greta Moorkens, G., Hans, G. and De Clerck, L.S. In the mind or in the brain? Scientific evidence for central sensitisation in chronic fatigue syndrome. European Journal of Clinical Investigations. 2012; 42 (2): 203-212; Tanaka, M. and Watanabe, Y. A new hypothesis of chronic fatigue syndrome: Co-conditioning theory. Medical Hypotheses 75 2010; 244-249; Kadotaa, Y., Cooperb, G., Burtona, A.R., Lemona, J., Schallb,U., Lloyde, A. and Vollmer-Connaa,U. Autonomic hyper-vigilance in postinfective fatigue syndrome. Biological Psychology 85 2010; 97-103; Knoopa, H., Prinsb, J.B., Moss-Morrisc, R. and Bleijenbergd, G. The central role of cognitive processes in the perpetuation of chronic fatigue syndrome. Journal of Psychosomatic Research 68 2010; 489-494; Wiborg, J.F., Knoop, H., Frank, L.E. and Bleijenberg, G. Towards an evidencebased treatment model for cognitive behavioral interventions focusing on chronic fatigue syndrome. Journal of Psychosomatic Research 72 2012; 399-404; and Friedberg., F. Chronic Fatigue Syndrome, Fibromyalgia, and Related Illnesses: A Clinical Model of Assessment and Intervention. Journal of Clinical Psychology 2010; 66:641-665.

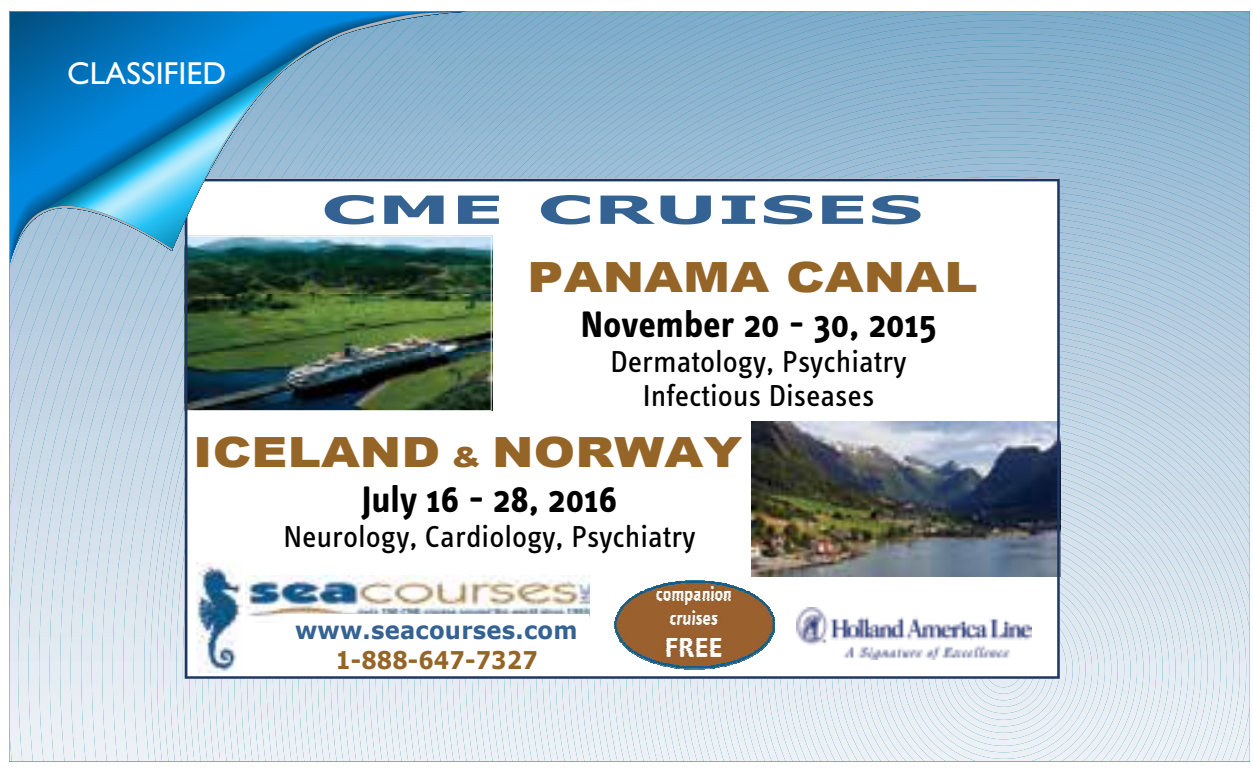

